\title{
Introduction to Random Signals and Noise
}




\title{
Introduction to \\ Random Signals and Noise
}

\author{
Wim C. van Etten
}

University of Twente, The Netherlands

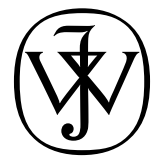

John Wiley \& Sons, Ltd 
Copyright (C) 2005 John Wiley \& Sons Ltd, The Atrium, Southern Gate, Chichester, West Sussex PO19 8SQ, England

Telephone (+44) 1243779777

Email (for orders and customer service enquiries): cs-books@wiley.co.uk Visit our Home Page on www.wiley.com

All Rights Reserved. No part of this publication may be reproduced, stored in a retrieval system or transmitted in any form or by any means, electronic, mechanical, photocopying, recording, scanning or otherwise, except under the terms of the Copyright, Designs and Patents Act 1988 or under the terms of a licence issued by the Copyright Licensing Agency Ltd, 90 Tottenham Court Road, London W1T 4LP, UK, without the permission in writing of the Publisher. Requests to the Publisher should be addressed to the Permissions Department, John Wiley \& Sons Ltd, The Atrium, Southern Gate, Chichester, West Sussex PO19 8SQ, England, or emailed to permreq@wiley.co.uk, or faxed to (+44) 1243770620.

Designations used by companies to distinguish their products are often claimed as trademarks. All brand names and product names used in this book are trade names, service marks, trademarks or registered trademarks of their respective owners. The Publisher is not associated with any product or vendor mentioned in this book.

This publication is designed to provide accurate and authoritative information in regard to the subject matter covered. It is sold on the understanding that the Publisher is not engaged in rendering professional services. If professional advice or other expert assistance is required, the services of a competent professional should be sought.

\section{Other Wiley Editorial Offices}

John Wiley \& Sons Inc., 111 River Street, Hoboken, NJ 07030, USA

Jossey-Bass, 989 Market Street, San Francisco, CA 94103-1741, USA

Wiley-VCH Verlag GmbH, Boschstr. 12, D-69469 Weinheim, Germany

John Wiley \& Sons Australia Ltd, 42 McDougall Street, Milton, Queensland 4064, Australia

John Wiley \& Sons (Asia) Pte Ltd, 2 Clementi Loop \# 02-01, Jin Xing Distripark, Singapore 129809

John Wiley \& Sons Canada Ltd, 22 Worcester Road, Etobicoke, Ontario, Canada M9W 1L1

Wiley also publishes its books in a variety of electronic formats. Some content that appears in print may not be available in electronic books.

\section{British Library Cataloguing in Publication Data}

A catalogue record for this book is available from the British Library

ISBN-13 978-0-470-02411-9 (HB)

ISBN-10 0-470-02411-9 (HB)

Typeset in 10/12pt Times by Thomson Press (India) Limited, New Delhi Printed and bound in Great Britain by Antony Rowe Ltd, Chippenham, Wiltshire This book is printed on acid-free paper responsibly manufactured from sustainable forestry in which at least two trees are planted for each one used for paper production. 
To Kitty,

to Sascha, Anne and Emmy,

to Björn and Esther 


\section{Contents}

Preface $\quad$ xi

1 Introduction $\quad 1$

1.1 Random Signals and Noise 1

1.2 Modelling 1

1.3 The Concept of a Stochastic Process 2

1.3.1 Continuous Stochastic Processes 4

1.3.2 Discrete-Time Processes (Continuous Random Sequences) 5

1.3.3 Discrete Stochastic Processes 6

$\begin{array}{lll}\text { 1.3.4 Discrete Random Sequences } & 7\end{array}$

1.3.5 Deterministic Function versus Stochastic Process 8

1.4 Summary 8

2 Stochastic Processes 9

2.1 Stationary Processes 9

2.1.1 Cumulative Distribution Function and Probability Density Function 9

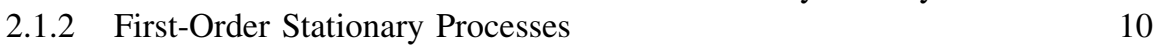

2.1.3 Second-Order Stationary Processes 11

2.1.4 Nth-Order Stationary Processes 11

2.2 Correlation Functions 11

2.2.1 The Autocorrelation Function, Wide-Sense Stationary
Processes and Ergodic Processes

2.2.2 Cyclo-Stationary Processes 16

2.2.3 The Cross-Correlation Function 19

2.2.4 Measuring Correlation Functions 24

2.2.5 Covariance Functions 26

2.2.6 Physical Interpretation of Process Parameters 27

$\begin{array}{lll}2.3 & \text { Gaussian Processes } & 27\end{array}$

2.4 Complex Processes 30

2.5 Discrete-Time Processes 31

2.5.1 Mean, Correlation Functions and Covariance Functions 31

$\begin{array}{lll}2.6 & \text { Summary } & 33\end{array}$

2.7 Problems 34

3 Spectra of Stochastic Processes $\quad 39$

3.1 The Power Spectrum 39 
3.2 The Bandwidth of a Stochastic Process 43

3.3 The Cross-Power Spectrum 45

3.4 Modulation of Stochastic Processes 47

3.4.1 Modulation by a Random Carrier $\quad 49$

3.5 Sampling and Analogue-To-Digital Conversion $\quad 50$

3.5.1 Sampling Theorems 51

3.5.2 A/D Conversion $\quad 54$

3.6 Spectrum of Discrete-Time Processes $\quad 57$

$\begin{array}{lll}3.7 & \text { Summary } & 58\end{array}$

$\begin{array}{lll}3.8 & \text { Problems } & 59\end{array}$

4. Linear Filtering of Stochastic Processes $\quad 65$

4.1 Basics of Linear Time-Invariant Filtering 65

4.2 Time Domain Description of Filtering of Stochastic Processes 68

$\begin{array}{ll}\text { 4.2.1 The Mean Value of the Filter Output } & 68\end{array}$

4.2.2 The Autocorrelations Function of the Output 69

$\begin{array}{ll}\text { 4.2.3 Cross-Correlation of the Input and Output } & 70\end{array}$

4.3 Spectra of the Filter Output 71

4.4 Noise Bandwidth $\quad 74$

$\begin{array}{lll}\text { 4.4.1 Band-Limited Processes and Systems } & 74\end{array}$

$\begin{array}{lll}\text { 4.4.2 Equivalent Noise Bandwidth } & 75\end{array}$

4.5 Spectrum of a Random Data Signal 77

4.6 Principles of Discrete-Time Signals and Systems 82

$\begin{array}{ll}\text { 4.6.1 The Discrete Fourier Transform } & 82\end{array}$

4.6.2 The $z$-Transform $\quad 86$

4.7 Discrete-Time Filtering of Random Sequences 90

4.7.1 Time Domain Description of the Filtering 90

4.7.2 Frequency Domain Description of the Filtering 91

$\begin{array}{lll}4.8 & \text { Summary } & 93\end{array}$

4.9 Problems 94

5 Bandpass Processes $\quad 101$

5.1 Description of Deterministic Bandpass Signals 101

5.2 Quadrature Components of Bandpass Processes 106

5.3 Probability Density Functions of the Envelope and 111

$\begin{array}{ll}5.4 \text { Measurement of Spectra } & 115\end{array}$

$\begin{array}{lll}\text { 5.4.1 The Spectrum Analyser } & 115\end{array}$

$\begin{array}{ll}\text { 5.4.2 Measurement of the Quadrature Components } & 118\end{array}$

$\begin{array}{lll}5.5 & \text { Sampling of Bandpass Processes } & 119\end{array}$

$\begin{array}{ll}\text { 5.5.1 Conversion to Baseband } & 119\end{array}$

$\begin{array}{lr}\text { 5.5.2 Direct Sampling } & 119\end{array}$

$\begin{array}{lll}5.6 & \text { Summary } & 121\end{array}$

5.7 Problems 121

6 Noise in Networks and Systems 129

$\begin{array}{ll}6.1 & 129\end{array}$

6.2 Thermal Noise in Resistors 130

6.3 Thermal Noise in Passive Networks 131 
$\begin{array}{lll}6.4 & \text { System Noise } & 137\end{array}$

$\begin{array}{lll}6.4 .1 & \text { Noise in Amplifiers } & 138\end{array}$

$\begin{array}{lll}6.4 .2 & \text { The Noise Figure } & 140\end{array}$

$\begin{array}{ll}\text { 6.4.3 Noise in Cascaded systems } & 142\end{array}$

$\begin{array}{lll}6.5 & \text { Summary } & 146\end{array}$

6.6 Problems 146

7 Detection and Optimal Filtering $\quad 153$

$\begin{array}{llr}7.1 & \text { Signal Detection } & 154\end{array}$

$\begin{array}{lll}\text { 7.1.1 Binary Signals in Noise } & 154\end{array}$

7.1.2 Detection of Binary Signals in White Gaussian Noise 158

7.1.3 Detection of $M$-ary Signals in White Gaussian Noise 161

\begin{tabular}{ll}
7.1 .4 & 165 \\
\hline
\end{tabular}

7.2 Filters that Maximize the Signal-to-Noise Ratio 165

$\begin{array}{lll}7.3 & \text { The Correlation Receiver } & 171\end{array}$

7.4 Filters that Minimize the Mean-Squared Error $\quad 175$

$\begin{array}{ll}\text { 7.4.1 The Wiener Filter Problem } & 175\end{array}$

$\begin{array}{lll}7.4 .2 & \text { Smoothing } & 176\end{array}$

$\begin{array}{lll}7.4 .3 & \text { Prediction } & 179\end{array}$

$\begin{array}{lr}\text { 7.4.4 Discrete-Time Wiener Filtering } & 183\end{array}$

$\begin{array}{llr}7.5 & \text { Summary } & 185\end{array}$

$\begin{array}{lll}7.6 & \text { Problems } & 185\end{array}$

8 Poisson Processes and Shot Noise 193

$\begin{array}{lll}8.1 & \text { Introduction } & 193\end{array}$

8.2 The Poisson Distribution 194

$\begin{array}{lll}\text { 8.2.1 The Characteristic Function } & 194\end{array}$

$\begin{array}{lll}\text { 8.2.2 Cumulants } & 196\end{array}$

$\begin{array}{ll}\text { 8.2.3 Interarrival Time and Waiting Time } & 197\end{array}$

$\begin{array}{ll}\text { 8.3 The Homogeneous Poisson Process } & 198\end{array}$

8.3.1 Filtering of Homogeneous Poisson Processes and Shot Noise 199

$\begin{array}{ll}\text { 8.4 Inhomogeneous Poisson Processes } & 204\end{array}$

8.5 The Random-Pulse Process 205

$\begin{array}{lll}8.6 & \text { Summary } & 207\end{array}$

8.7 Problems 208

$\begin{array}{ll}\text { References } & 211\end{array}$

Further Reading $\quad 213$

$\begin{array}{ll}\text { Appendices } & 215\end{array}$

A. Representation of Signals in a Signal Space $\quad 215$

$\begin{array}{lll}\text { A.1 Linear Vector Spaces } & 215\end{array}$

$\begin{array}{ll}\text { A.2 The Signal Space Concept } & 216\end{array}$

$\begin{array}{ll}\text { A.3 Gram-Schmidt Orthogonalization } & 218\end{array}$

A.4 The Representation of Noise in Signal Space 219

$\begin{array}{ll}\text { A.4.1 Relevant and Irrelevant Noise } & 221\end{array}$

$\begin{array}{lll}\text { A.5 Signal Constellations } & 222\end{array}$

$\begin{array}{lll}\text { A.5.1 Binary Antipodal Signals } & 222\end{array}$ 
A.5.2 Binary Orthogonal Signals

A.5.3 Multiphase Signals

A.5.4 Multiamplitude Signals

A.5.5 QAM Signals

A.5.6 $M$-ary Orthogonal Signals

A.5.7 Biorthogronal Signals

A.5.8 Simplex Signals

A.6 Problems

B. Attenuation, Phase Shift and Decibels 229

C. Mathematical Relations 231

C.1 Trigonometric Relations 231

$\begin{array}{lll}\text { C.2 Derivatives } & 232\end{array}$

C.2.1 Rules fn Differentiation 232

$\begin{array}{lll}\text { C.2.1 Chain Rule } & 232\end{array}$

C.2.3 Stationary Points 233

C.3 Indefinite Integrals 233

$\begin{array}{lll}\text { C.3.1 Basic Integrals } & 233\end{array}$

$\begin{array}{lll}\text { C.3.2 Integration by Parts } & 234\end{array}$

C.3.3 Rational Algebraic Functions 234

C.3.4 Trigonometric Functions 235

$\begin{array}{ll}\text { C.3.5 Exponential Functions } & 236\end{array}$

$\begin{array}{lll}\text { C.4 Definite Integrals } & 236\end{array}$

$\begin{array}{lll}\text { C.5 } & \text { Series } & 237\end{array}$

$\begin{array}{lll}\text { C.6 Logarithms } & 238\end{array}$

D. Summary of Probability Theory 239

E. Definition of a Few Special Functions

F. The $Q($.$) and erfc Function \quad 243$

G. Fourier Transforms $\quad 245$

H. Mathematical and Physical Constants $\quad 247$

$\begin{array}{ll}\text { Index } & 249\end{array}$ 


\section{Preface}

Random signals and noise are present in several engineering systems. Practical signals seldom lend themselves to a nice mathematical deterministic description. It is partly a consequence of the chaos that is produced by nature. However, chaos can also be man-made, and one can even state that chaos is a conditio sine qua non to be able to transfer information. Signals that are not random in time but predictable contain no information, as was concluded by Shannon in his famous communication theory.

To deal with this randomness we have to nevertheless use a characterization in deterministic terms; i.e. we employ probability theory to determine characteristic descriptions such as mean, variance, correlation, etc. Whenever chaotic behaviour is timedependent, as is often the case for random signals, the time parameter comes into the picture. This calls for an extension of probability theory, which is the theory of stochastic processes and random signals. With the involvement of time, the phenomenon of frequency also enters the picture. Consequently, random signal theory leans heavily on both probability and Fourier theories. Combining these subjects leads to a powerful tool for dealing with random signals and noise.

In practice, random signals may be encountered as a desired signal such as video or audio, or it may be an unwanted signal that is unintentionally added to a desired (information bearing) signal thereby disturbing the latter. One often calls this unwanted signal noise. Sometimes the undesired signal carries unwanted information and does not behave like noise in the classical sense. In such cases it is termed as interference. While it is usually difficult to distinguish (at least visually) between the desired signal and noise (or interference), by means of appropriate signal processing such a distinction can be made. For example, optimum receivers are able to enhance desired signals while suppressing noise and interference at the same time. In all cases a description of the signals is required in order to be able to analyse their impact on the performance of the system under consideration. In communication theory this situation often occurs. The random time-varying character of signals is usually difficult to describe, and this is also true for associated signal processing activities such as filtering. Nevertheless, there is a need to characterize these signals using a few deterministic parameters that allow a system user to assess system performance.

This book deals with stochastic processes and noise at an introductory level. Probability theory is assumed to be known. The same holds for mathematical background in differential and integral calculus, Fourier analysis and some basic knowledge of network and linear system theory. It introduces the subject in the form of theorems, properties and examples. Theorems and important properties are placed in frames, so that the student can easily 
summarize them. Examples are mostly taken from practical applications. Each chapter concludes with a summary and a set of problems that serves as practice material. The book is well suited for dealing with the subject at undergraduate level. A few subjects can be skipped if they do not fit into a certain curriculum. Besides, the book can also serve as a reference for the experienced engineer in his daily work.

In Chapter 1 the subject is introduced and the concept of a stochastic process is presented. Different types of processes are defined and elucidated by means of simple examples.

Chapter 2 gives the basic definitions of probability density functions and includes the time dependence of these functions. The approach is based on the 'ensemble' concept. Concepts such as stationarity, ergodicity, correlation functions and covariance functions are introduced. It is indicated how correlation functions can be measured. Physical interpretation of several stochastic concepts are discussed. Cyclo-stationary and Gaussian processes receive extra attention, as they are of practical importance and possess some interesting and convenient properties. Complex processes are defined analogously to complex variables. Finally, the different concepts are reconsidered for discrete-time processes.

In Chapter 3 a description of stochastic processes in the frequency domain is given. This results in the concept of power spectral density. The bandwidth of a stochastic process is defined. Such an important subject as modulation of stochastic processes is presented, as well as the synchronous demodulation. In order to be able to define and describe the spectrum of discrete-time processes, a sampling theorem for these processes is derived.

After the basic concepts and definitions treated in the first three chapters, Chapter 4 starts with applications. Filtering of stochastic processes is the main subject of this chapter. We confine ourselves to linear, time-invariant filtering and derive both the correlation functions and spectra of a two-port system. The concept of equivalent noise bandwidth has been defined in order to arrive at an even more simple description of noise filtering in the frequency domain. Next, the calculation of the spectrum of random data signals is presented. A brief resumé of the principles of discrete-time signals and systems is dealt with using the $z$-transform and discrete Fourier transform, based on which the filtering of discrete-time processes is described both in time and frequency domains.

Chapter 5 is devoted to bandpass processes. The description of bandpass signals and systems in terms of quadrature components is introduced. The probability density functions of envelope and phase are derived. The measurement of spectra and operation of the spectrum analyser is discussed. Finally, sampling and conversion to baseband of bandpass processes is discussed.

Thermal noise and its impact on systems is the subject of Chapter 6. After presenting the spectral densities we consider the role of thermal noise in passive networks. System noise is considered based on the thermal noise contribution of amplifiers, the noise figure and the influence of cascading of systems on noise performance.

Chapter 7 is devoted to detection and optimal filtering. The chapter starts by considering hypothesis testing, which is applied to the detection of a binary signal disturbed by white Gaussian noise. The matched filter emerges as the optimum filter for optimum detection performance. Finally, filters that minimize the mean squared error (Wiener filters) are derived. They can be used for smoothing stored data or portions of a random signal that arrived in the past. Filters that produce an optimal prediction of future signal values can also be designed.

Finally, Chapter 8 is of a more advanced nature. It presents the basics of random point processes, of which the Poisson process is the most well known. The characteristic function 
plays a crucial role in analysing these processes. Starting from that process several shot noise processes are introduced: the homogeneous Poisson process, the inhomogeneous Poisson process, the Poisson impulse process and the random-pulse process. Campbell's theorem is derived. A few application areas of random point processes are indicated.

The appendices contain a few subjects that are necessary for the main material. They are: signal space representation and definitions of attenuation, phase shift and decibels. The rest of the appendices comprises basic mathematical relations, a summary of probability theory, definitions of special functions, a list and properties of Fourier transform pairs, and a few mathematical and physical constants.

Finally, I would like to thank those people who contributed in one way or another to this text. My friend Rajan Srinivasan provided me with several suggestions to improve the content. Also, Arjan Meijerink carefully read the draft and made suggestions for improvement.

Last but certainly not least, I thank my wife Kitty, who allowed me to spend so many hours of our free time to write this text.

Wim van Etten

Enschede, The Netherlands 


\section{1}

\section{Introduction}

\subsection{RANDOM SIGNALS AND NOISE}

In (electrical) engineering one often encounters signals that do not have a precise mathematical description, since they develop as random functions of time. Sometimes this random development is caused by a single random variable, but often it is a consequence of many random variables. In other cases the causes of randomness are not clear and a description is not possible, but the signal is characterized by means of measurements only.

A random time function may be a desired signal, such as an audio or video signal, or it may be an unwanted signal that is unintentionally added to a desired (information) signal and disturbs the desired signal. We call the desired signal a random signal and the unwanted signal noise. However, the latter often does not behave like noise in the classical sense, but it is more like interference. Then it is an information bearing signal as well, but undesired. A desired signal and noise (or interference) can, in general, not be distinguished completely; by means of well-defined signal processing in a receiver, the desired signal may be favoured in a maximal way whereas the disturbance is suppressed as much as possible. In all cases a description of the signals is required in order to be able to analyse its impact on the performance of the system under consideration. Especially in communication theory this situation often occurs. The random character as a function of time makes the signals difficult to describe and the same holds for signal processing or filtering. Nevertheless, there is a need to characterize these signals by a few deterministic parameters that enable the system user to assess the performance of the system. The tool to deal with both random signals and noise is the concept of the stochastic process, which is introduced in Section 1.3.

This book gives an elementary introduction to the methods used to describe random signals and noise. For that purpose use is made of the laws of probability, which are extensively described in textbooks [1-5].

\subsection{MODELLING}

When studying and analysing random signals one is mainly committed to theory, which however, can be of good predictive value. Actually, the main activity in the field of random signals is modelling of processes and systems. Many scientists and engineers have

Introduction to Random Signals and Noise W. van Etten (C) 2005 John Wiley \& Sons, Ltd 


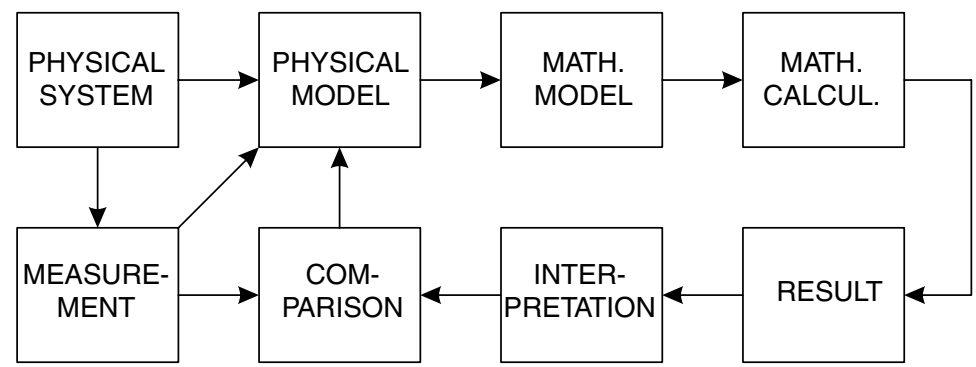

Figure 1.1 The process of modelling

contributed to that activity in the past and their results have been checked in practice. When a certain result agrees (at least to a larger extent) with practical measurements, then there is confidence in and acceptance of the result for practical application. This process of modelling has schematically been depicted in Figure 1.1.

In the upper left box of this scheme there is the important physical process. Based on our knowledge of the physics of this process we make a physical model of it. This physical model is converted into a mathematical model. Both modelling activities are typical engineer tasks. In this mathematical model the physics is no longer formally recognized, but the laws of physics will be included with their mathematical description. Once the mathematical model has been completed and the questions are clear we can forget about the physics for the time being and concentrate on doing the mathematical calculations, which may help us to find the answers to our questions. In this phase the mathematicians can help the engineer a lot. Let us suppose that the mathematical calculations give a certain outcome, or maybe several outcomes. These outcomes would then need to be interpreted in order to discover what they mean from a physical point of view. This ends the role of the mathematician, since this phase is maybe the most difficult engineering part of the process. It may happen that certain mathematical solutions have to be discarded since they contradict physical laws. Once the interpretation has been completed there is a return to the physical process, as the practical applicability of the results needs to be checked. In order to check these the quantities or functions that have been calculated are measured. The measurement is compared to the calculated result and in this way the physical model is validated. This validation may result in an adjustment of the physical model and another cycle in the loop is made. In this way the model is refined iteratively until we are satisfied about the validation. If there is a shortage of insight into the physical system, so that the physical model is not quite clear, measurements of the physical system may improve the physical model.

In the courses that are taught to students, models that have mainly been validated in this way are presented. However, it is important that students are aware of this process and the fact that the models that are presented may be a result of a difficult struggle for many years by several physicists, engineers and mathematicians. Sometimes students are given the opportunity to be involved in this process during research assignments.

\subsection{THE CONCEPT OF A STOCHASTIC PROCESS}

In probability theory a random variable is a rule that assigns a number to every outcome of an experiment, such as, for example, rolling a die. This random variable $X$ is associated with a sample space $S$, such that according to a well-defined procedure to each event $s$ in the 


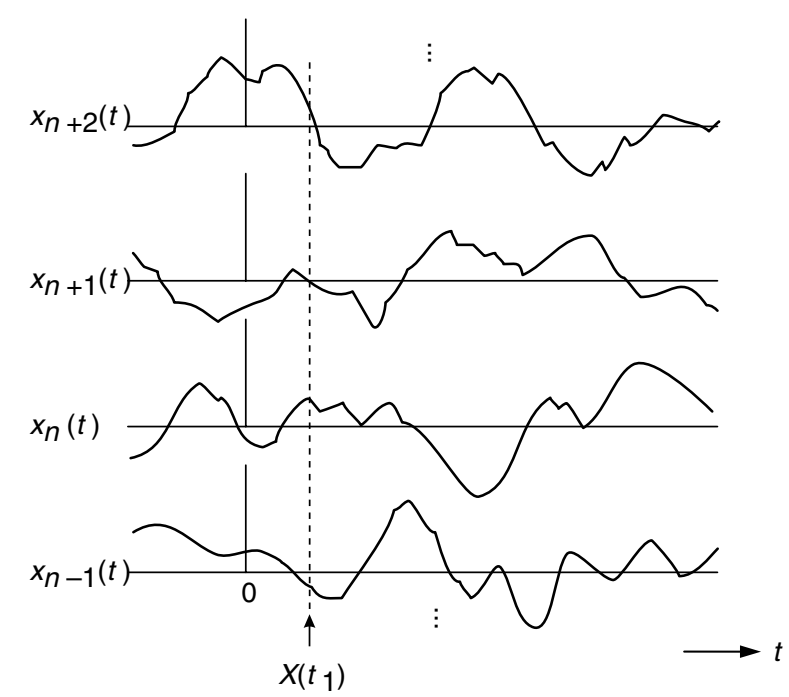

Figure 1.2 A few sample functions of a stochastic process

sample space a number is assigned to $X$ and is denoted by $X(s)$. For stochastic processes, on the other hand, a time function $x(t, s)$ is assigned to every outcome in the sample space. Within the framework of the experiment the family (or ensemble) of all possible functions that can be realized is called the stochastic process and is denoted by $X(t, s)$. A specific waveform out of this family is denoted by $x_{n}(t)$ and is called a sample function or a realization of the stochastic process. When a realization in general is indicated the subscript $n$ is omitted. Figure 1.2 shows a few sample functions that are supposed to constitute an ensemble. The figure gives an example of a finite number of possible realizations, but the ensemble may consist of an infinite number of realizations. The realizations may even be uncountable. A realization itself is sometimes called a stochastic process as well. Moreover, a stochastic process produces a random variable that arises from giving $t$ a fixed value with $s$ being variable. In this sense the random variable $X\left(t_{1}, s\right)=X\left(t_{1}\right)$ is found by considering the family of realizations at the fixed point in time $t_{1}$ (see Figure 1.2). Instead of $X\left(t_{1}\right)$ we will also use the notation $X_{1}$. The random variable $X_{1}$ describes the statistical properties of the process at the instant of time $t_{1}$. The expectation of $X_{1}$ is called the ensemble mean or the expected value or the mean of the stochastic process (at the instant of time $t_{1}$ ). Since $t_{1}$ may be arbitrarily chosen, the mean of the process will in general not be constant, i.e. it may have different values for different values of $t$. Finally, a stochastic process may represent a single number by giving both $t$ and $s$ fixed values. The phrase 'stochastic process' may therefore have four different interpretations. They are:

1. A family (or ensemble) of time functions. Both $t$ and $s$ are variables.

2. A single time function called a sample function or a realization of the stochastic process. Then $t$ is a variable and $s$ is fixed.

3. A random variable; $t$ is fixed and $s$ is variable.

4. A single number; both $t$ and $s$ are fixed. 
Which of these four interpretations holds in a specific case should follow from the context.

Different classes of stochastic processes may be distinguished. They are classified on the basis of the characteristics of the realization values of the process $x$ and the time parameter $t$. Both can be either continuous or discrete, in any combination. Based on this we have the following classes:

- Both the values of $X(t)$ and the time parameter $t$ are continuous. Such a process is called a continuous stochastic process.

- The values of $X(t)$ are continuous, whereas time $t$ is discrete. These processes are called discrete-time processes or continuous random sequences. In the remainder of the book we will use the term discrete-time process.

- If the values of $X(t)$ are discrete but the time axis is continuous, we call the process a discrete stochastic process.

- Finally, if both the process values and the time scale are discrete, we say that the process is a discrete random sequence.

In Table 1.1 an overview of the different classes of processes is presented. In order to get some feeling for stochastic processes we will consider a few examples.

Table 1.1 Summary of names of different processes

\begin{tabular}{l|ll}
\hline \multirow{2}{*}{$X(t)$} & \multicolumn{2}{|c}{ Time } \\
\cline { 2 - 3 } & Continuous & Discrete \\
\hline Continuous & Continuous stochastic process & Discrete-time process \\
Discrete & Discrete stochastic process & Discrete random sequence \\
\hline
\end{tabular}

\subsubsection{Continuous Stochastic Processes}

For this class of processes it is assumed that in principle the following holds:

$$
-\infty<x(t)<\infty \text { and }-\infty<t<\infty
$$

An example of this class was already given by Figure 1.2. This could be an ensemble of realizations of a thermal noise process as is, for instance, produced by a resistor, the characteristics of which are to be dealt with in Chapter 6. The underlying experiment is selecting a specific resistor from a collection of, let us say, $100 \Omega$ resistors. The voltage across every selected resistor corresponds to one of the realizations in the figure.

Another example is given below.

\section{Example 1.1:}

The process we consider now is described by the equation

$$
X(t)=\cos \left(\omega_{0} t-\Theta\right)
$$




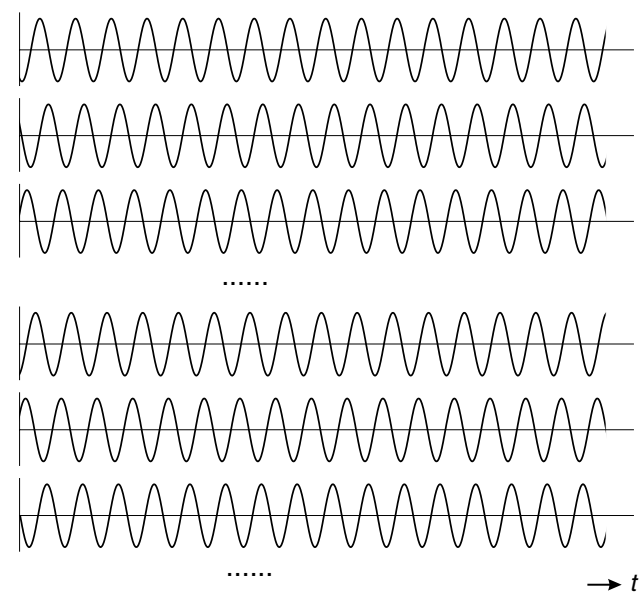

Figure 1.3 Ensemble of sample functions of the stochastic process $\cos \left(\omega_{0} t-\Theta\right)$, with $\Theta$ uniformly distributed on the interval $(0,2 \pi]$

with $\omega_{0}$ a constant and $\Theta$ a random variable with a uniform probability density function on the interval $(0,2 \pi]$. In this example the set of realizations is in fact uncountable, as $\Theta$ assumes continuous values. The ensemble of sample functions is depicted in Figure 1.3.

Thus each sample function consists of a cosine function with unity amplitude, but the phase of each sample function differs randomly from others. For each sample function a drawing is taken from the uniform phase distribution. We can imagine this process as follows. Consider a production process of crystal oscillators, all producing the same amplitude unity and the same radial frequency $\omega_{0}$. When all those oscillators are switched on, their phases will be mutually independent. The family of all measured output waveforms can be considered as the ensemble that has been presented in Figure 1.3.

This process will get further attention in different chapters that follow.

\subsubsection{Discrete-Time Processes (Continuous Random Sequences)}

The description of this class of processes becomes more and more important due to the increasing use of modern digital signal processors which offer flexibility and increasing speed and computing power. As an example of a discrete-time process we can imagine sampling the process that was given in Figure 1.2. Let us suppose that to this process ideal sampling is applied at equidistant points in time with sampling period $T_{\mathrm{s}}$; with ideal sampling we mean the sampling method where the values at $T_{\mathrm{s}}$ are replaced by delta functions of amplitude $X\left(n T_{\mathrm{s}}\right)$ [6]. However, to indicate that it is now a discrete-time process we denote it by $X[n]$, where $n$ is an integer running in principle from $-\infty$ to $+\infty$. We know from the sampling theorem (see Section 3.5.1 or, for instance, references [1] and [7]) that the original signal can perfectly be recovered from its samples, provided that the signals are band-limited. The process that is produced in this way is given in Figure 1.4, where the sample values are presented by means of the length of the arrows. 


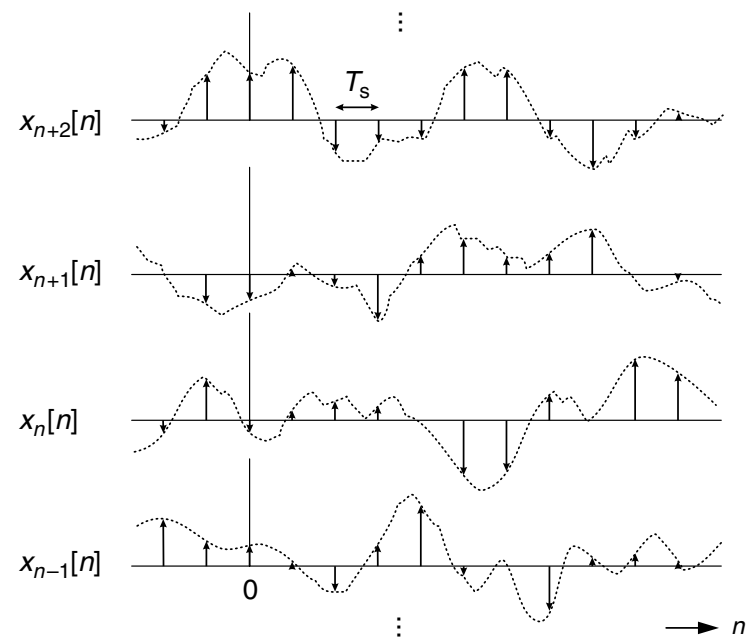

Figure 1.4 Example of a discrete-time stochastic process

Another important example of the discrete-time process is the so-called Poisson process, where there are no equidistant samples in time but the process produces 'samples' at random points in time. This process is an adequate model for shot noise and it is dealt with in Chapter 8 .

\subsubsection{Discrete Stochastic Processes}

In this case the time is continuous and the values discrete. We present two examples of this class. The second one, the random data signal, is of great practical importance and we will consider it in further detail in Chapter 4.

\section{Example 1.2:}

This example is a very simple one. The ensemble of realizations consists of a set of constant time functions. According to the outcome of an experiment one of these constants may be chosen. This experiment can be, for example, the rolling of a die. In that case the number of realizations can be six $(n=6)$, equal to the usual number of faces of a die. Each of the outcomes $s \in\{1,2,3,4,5,6\}$ has a one-to-one correspondence to one of these numbered constant functions of time. The ensemble is depicted in Figure 1.5.

\section{Example 1.3:}

Another important stochastic process is the random data signal. It is a signal that is produced by many data sources and is described by

$$
X(t)=\sum_{n} A_{n} p(t-n T-\Theta)
$$




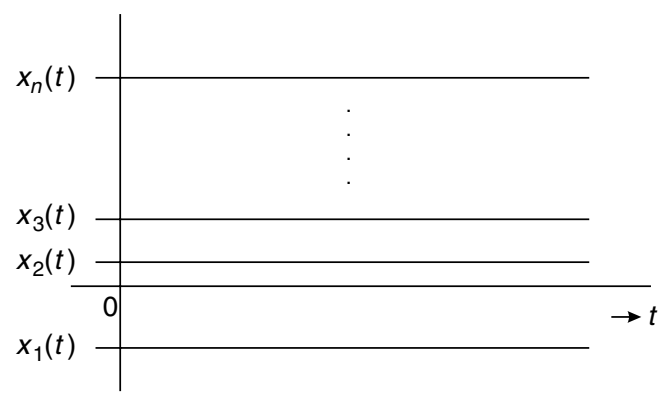

Figure 1.5 Ensemble of sample functions of the stochastic process constituted by a number of constant time functions

where $\left\{A_{n}\right\}$ are the data bits that are randomly chosen from the set $A_{n} \in\{+1,-1\}$. The rectangular pulse $p(t)$ of width $T$ serves as the carrier of the information. Now $\Theta$ is supposed to be uniformly distributed on the bit interval $(0, T]$, so that all data sources of the family have the same bit period, but these periods are not synchronized. The ensemble is given in Figure 1.6.

\subsubsection{Discrete Random Sequences}

The discrete random sequence can be imagined to result from sampling a discrete stochastic process. Figure 1.7 shows the result of sampling the random data signal from Example 1.3.

We will base the further development of the concept, description and properties of stochastic processes on the continuous stochastic process. Then we will show how these are extended to discrete-time processes. The two other classes do not get special attention, but

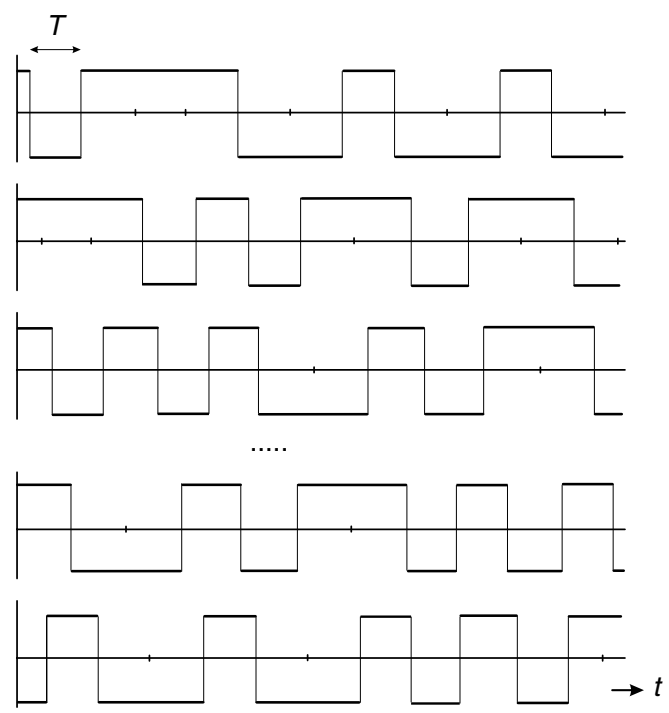

Figure 1.6 Ensemble of sample functions of the stochastic process $\sum_{n} A_{n} p(t-n T-\Theta)$, with $\Theta$ uniformly distributed on the interval $(0, T]$ 


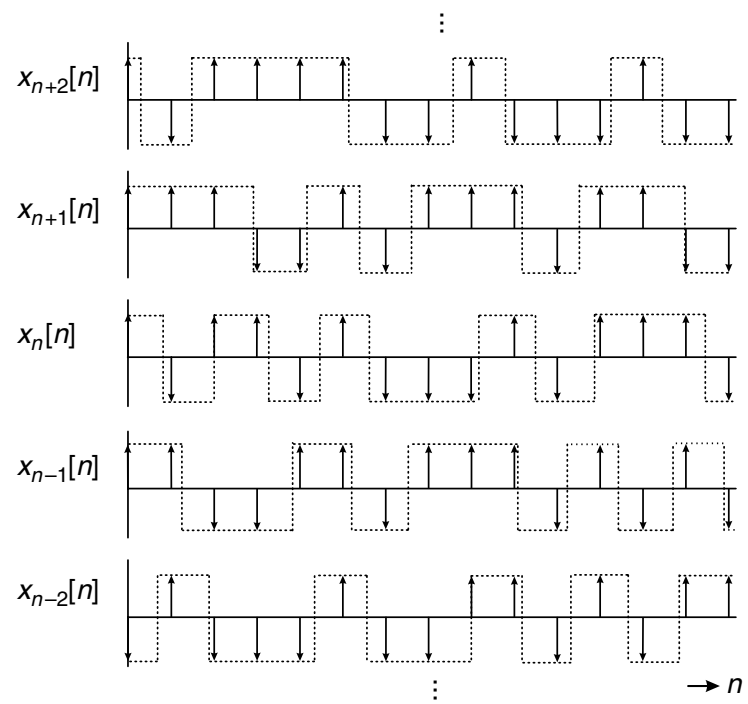

Figure 1.7 Example of a discrete random sequence

are considered as special cases of the former ones by limiting the realization values $x$ to a discrete set.

\subsubsection{Deterministic Function versus Stochastic Process}

The concept of the stochastic process does not conflict with the theory of deterministic functions. It should be recognized that a deterministic function can be considered as nothing else but a special case of a stochastic process. This is elucidated by considering Example 1.1. If the random variable $\Theta$ is given the probability density function $f_{\Theta}(\theta)=\delta(\theta)$, then the stochastic process reduces to the function $\cos \left(\omega_{0} t\right)$. The given probability density function is actually a discrete one with a single outcome. In fact, the ensemble of the process reduces in this case to a family comprising merely one member. This is a general rule; when the probability density function of the stochastic process that is governed by a single random variable consists of a single delta function, then a deterministic function results. This way of generalization avoids the often confusing discussion on the difference between a deterministic function on the one hand and a stochastic process on the other hand. In view of the consideration presented here they can actually be considered as members of the same class, namely the class of stochastic processes.

\subsection{SUMMARY}

Definitions of random signals and noise have been given. A random signal is, as a rule, an information carrying wanted signal that behaves randomly. Noise also behaves randomly but is unwanted and disturbs the signal. A common tool to describe both is the concept of a stochastic process. This concept has been explained and different classes of stochastic processes have been identified. They are distinguished by the behaviour of the time parameter and the values of the process. Both can either be continuous or discrete. 


\section{Index}

Symbols

$\delta$-function, 5, 67, 78, 81, 83, 205, 242

discrete-time -, 82

$\delta$-pulse, 51

a.c. component, 27

aliasing, 86

distortion, 52

amplifier

front-end -, 146

low-noise -, 146

optical -, 145

amplitude modulated (AM) signal, 101

amplitude shift keying (ASK), 112, 173,190

analog-to-digital conversion, 54

analytic

function, 180, 181

signal, 102

antenna noise, 144

anti-causal function, 180, 181

antipodal signals, 222

attenuation, 229

autocorrelation function, 11, 69

properties of -, 13

autocorrelation of discrete-time process, 32, 57, 90

autocovariance of discrete-time process, 32

available

power gain, 138

spectral density, 134

spectral density in amplifiers, 138

average

time -, 14 band-limited process

definition of -, 74

band-limiting filter definition of -, 74

bandpass

filter, 73

signal, 101

bandpass filtering of white noise, 110

bandpass process, 44 conversion to baseband, 119

definition of -, 74

direct sampling of -, 119

properties of -, 107

bandwidth

of bandpass process, 44

of low pass process, 43

root-mean-squared (r.m.s.) -, 43

baseband process

definition of -, 74

Bayes' theorem, 155

Bessel function, 113

biorthogonal signals, 225

binary detection

in Gaussian noise, 158

binary phase shift keying (BPSK), 191

bipolar nonreturn-to-zero (NRZ) signal, 98

bit error probability, 155, 157

Boltzmann's constant, 130, 247

Brownian motion, 130, 133

Butterworth filter, 98

Campbell's theorem, 201

extension of -, 204, 206

Introduction to Random Signals and Noise W. van Etten (C) 2005 John Wiley \& Sons, Ltd 
CATV networks, 225

causality, 68

chain rule, 232

characteristic frequency, 104, 112

characteristic function, 194, 204

joint -, 202

of shot noise, 201

second -, 194

second joint -, 202, 204

clutter, 207

code division multiple access (CDMA), 49

coloured noise, 130

complementary error-function, 158, 243

complete orthonormal set, 216

complex envelope, 102, 105, 111

spectrum of -, 105, 111

complex impulse response, 105

complex processes, 30, 111

applied to current, 31

applied to voltage, 31

conditional probabilities, 154, 155, 159, 163

constant resistance network, 151

constants

mathematical -, 247

physical -, 247

convolution, $67,68,160,245$

discrete -, 82, 90

correlation, 240

coefficient, 28

receiver, 171

correlation function, 11

measurement of -, 24

cost matrix, 165

covariance function

auto-, 26

cross-, 26

cross-correlation, 220, 222

of discrete-time process, 32,90

cross-correlation function, 19, 70

properties of -, 20

time averaged -, 21

cross-covariance of discrete-time process, 32

cross-power spectrum, 45

properties of -, 46

cumulant, 197

cumulative probability distribution function, 9

cyclo-stationary process, 16,77

data signal, 19, 77, 154, 157

autocorrelation function of -, 78

spectrum of -, 78, 79

d.c. component, 27,203 de-emphasis, 97

decibel, 118, 145, 229

list of - values, 230

decision

criterion, 165

regions, 155,156

statistics, 162

threshold, 155, 160

decision rule

Bayes -, 165

maximum a posteriori (MAP) -, 165

maximum likelihood (ML) -, 165

minimax -, 165

Neyman-Pearson -, 165

demodulation

coherent -, 48

synchronous -, 48, 118

derivatives, 232

detection

of binary signals, 154

optimum -, 162

differentiating network, 96

differentiation, 232

digital signal processor (DSP), 50, 184

discrete Fourier transform (DFT), 84

discrete-time

process, 5, 54, 193

signal, 82,86

system, 82,86

discrete-time Fourier transform (DTFT), 83

discriminator, 103

distance

measurement, 21

metric, 162

distortionless transmission, 121

distribution function, 9

diversity, 188

doubly stochastic process, 205

duality, 245

dynamic range, 55

effective noise temperature, 139

eigenfunctions, 67

electrons, 193, 198, 207

ensemble, 3

mean, 3

envelope, 102

detection, 106, 111

distribution, 113 
equalization, 99

equivalent

noise bandwidth, 75, 140

noise resistance, 134

noise temperature, 134, 138

equivalent baseband

system, 105

transfer function, 104

Erbium doped fiber amplifier (EDFA), 145

ergodic

jointly -, 21

ergodic process

definition of -, 14

ergodicity of discrete-time process, 33

error probability, 155, 164

estimation error, 176, 178

excess noise factor, 206

expansion

McLaurin -, 237

Taylor -, 237

expectation, 239

false alarm, 165

fast Fourier transform (FFT), 84

filter

non-recursive -, 88

recursive -, 89

tapped delay line -, 88

transversal -, 88

filtering

of processes, 68

of thermal noise, 135

finite impulse response (FIR) filter, 82, 89

FM

detection, 97

signal, 101

Fourier series, 57, 218

Fourier transform, 39, 57, 67, 71, 194

discrete -, 82, 84

properties of -, 245

table, 246

two-dimensional -, 202

frequency conversion, 49

frequency shift keying (FSK), 112, 161, 190

Friis' formulas, 143

front-end amplifier, 146

Gaussian noise, 158, 162, 163, 167, 219

Gaussian processes, 27, 72

bandpass -, 112 jointly -, 27

properties of -, 29

Gaussian random variables, 27

covariance matrix of -, 28

jointly -, 28

properties of -, 29

Gram-Schmidt orthogonalization, 218

group delay, 122

guard band, 62

harmonic signal, 218

Hermitian spectrum, 104

hypothesis testing, 154, 161

impulse response, 67, 68, 71, 87, 199, 201

complex -, 105

of matched filter, 161

of optimum filter, 167

independence, 239

independent processes, 10, 21

infinite impulse response (IIR) filter, 82, 89

information signal, 1, 205

inner product

of signals, 216

of vectors, 215

integrals

definite -, 236

indefinite -, 233

integrate-and-dump receiver, 175

interarrival time, 197

interpolation by sinc function, 52

intersymbol interference, 99

inverse discrete Fourier transform (IDFT), 84

inverse discrete-time Fourier transform

(IDTFT), 83

inverse fast Fourier transform (IFFT), 84

irrelevant noise, 160, 221

jitter, 175

Kronecker delta, 216

Laplace transform, 179

light emitting diode (LED), 50

likelihood ratio, 155

linear time-invariant (LTI)

filter, 65, 160, 201

system, 66

logarithms, 238

Lorentz profile, 42

low-noise amplifier, 146 
M-ary biorthogonal signals, 225

M-ary detection in Gaussian noise, 161

M-ary phase modulation, 224

Manchester code, 98

matched filter, 161, 162, 167

for coloured noise, 167

matched impedances, 138

matching network, 149

mathematical

constants, 247

relations, 231

maximum ratio combining, 189

McLaurin expansion, 237

mean

ensemble -, 3

frequency, 44

of discrete-time process, 31

value, 10

mean-squared error, 52, 178

minimization of -, 176

miss, 165

mixer, 117

modems

cable -, 225

telephone -, 225

modulation, 47

amplitude -, 101

by random carrier, 49

frequency -, 101

phase -, 102

moment generating function, 196

moments of random variable, 196

multiamplitude signals, 224

multiphase signals, 224

multiple-input multiple-output (MIMO) systems, 65

$$
\begin{aligned}
& \text { narrowband } \\
& \text { bandpass process } \\
& \text { definition of -, } 75 \\
& \text { system } \\
& \text { definition of -, } 74
\end{aligned}
$$

neper, 229

noise, 1

coloured -, 130

Gaussian bandpass -, 111

in optical amplifiers, 145

in systems, 137

multiplicative -, 201

presentation in signal space, 219 thermal -, 130

vector, 159,219

noise bandwidth

equivalent,- 76

noise figure

average,- 140

definition of -, 140

of a cable, 141

of an attenuator, 141

of cascade, 143

spot -, 140

standard -, 140

noise in amplifiers, 138

noise temperature

effective -, 139

of cascade, 143

system -, 143

noisy amplifier model, 139

nonreturn-to-zero (NRZ) signal

bipolar -, 98

polar -, 79, 98

norm of a vector, 215

Norton equivalent circuit, 131

Nyquist frequency, 51

Nyquist's theorem, 136

optical amplifier

Erbium doped fiber -, 145

semiconductor -, 145

optical signal detection, 207

optimum filter characteristic, 177

optimum smoothing filter, 178

orthogonal

processes, 21

quadrature processes, 109

random variables, 109

vectors, 215

orthogonal signals, 223

M-ary -, 225

orthonormal set, 158, 216

complete -, 216

orthonormal signals, 216

oscillator spectrum, 42

Parseval's formula, 168, 245

periodically stationary process, 16

phase

delay, 122

distribution, 5, 114, 115

shift, 229 
phase modulation

M-ary -, 224

phase reversal keying (PRK),

191

phase shift keying (PSK), 190

phasor, 102

photodetector, 198, 205

photodiode, 193, 194

avalanche -, 194, 207

photomultiplier tube, 194, 207

photons, 193, 198, 205

physical

constants, 247

interpretation, 27

Planck's constant, 130

Poisson

distribution, 193

impulse process, 194, 205

processes, 193

sum formula, 81

Poisson processes

homogeneous -, 193, 198

inhomogeneous -, 194, 204

sum of independent -, 196

polar nonreturn-to-zero (NRZ) signal, 79, 98

posterior probabilities, 165

power, 40

a.c.,- 27

electrical -, 133

in output process, 71

maximum transfer of -, 138, 147

of discrete-time process, 57

of stochastic process, 133

power spectrum, 39

cross-, 45

measurement of -, 116

properties of -, 40

pre-emphasis, 97

pre-envelope, 102

prediction, 175,179

discrete-time -, 184

pure,- 184

pure,- 179

prior probabilities, 154, 163, 165

probability density function, 10 , 239

Gaussian -, 27, 239

joint,- 10

Laplacian -, 186

Poisson -, 193 probability distribution function, 239

joint -

$N$ th-order -, 10

second-order -, 10

process

bandpass -, 48, 106

stationary -

$N$ th-order -, 11

first-order -, 10

second-order -, 11

Q-function, 158, 164, 243

quadrature

components, 102, 106, 118

measurement of -, 118

description of bandpass processes, 106

description of modulated signals, 101

processes, 107-109

signals, 218

quadrature amplitude modulated (QAM) signals, 225

quadrature phase shift keying (QPSK), 224

quantization, 54

characteristic, 55

error, 55

levels, 55

noise, 56

step size, 55

quantizer, 55

non-uniform -, 57

uniform -, 56

queuing, 197

radar, 207

detection, 165

ranging, 22

random

gain, 205

signal, 1

variable, 2

vector, 155

random data signal, 77, 154

spectrum of,- 78

random point processes, 193

random sequence

continuous -, 5

discrete -, 7

random-pulse process, 205

Rayleigh-distribution, 113

RC-network, 71, 76, 137

realization, 3 
reconstruction

of bandpass processes, 119

of sampled processes, 52

of sampled signals, 51

rectangular

function, 241

pulse, 79, 84, 201

rectifier, 103

relevant noise, 159, 221

return-to-zero signal unipolar -, 80

Rice-distribution, 113

root-mean-squared (r.m.s.)

bandwidth, 43

value, 27

sample function, 3

sampling, 5

direct -, 119

flat-top -, 62

ideal -, 51

of bandpass processes, 119

rate, $51,52,119$

theorem

for deterministic signals, 51

for stochastic processes, 52

Schwarz's inequality, 166, 216

semi-invariant, 197

semiconductor optical amplifier (SOA), 145

series, 237

shot noise, 199

signal

constellation, 219, 222

energy, 159, 162, 218

harmonic -, 218

restoration, 179

space, 161, 163, 216

vector, 159, 216

signal-to-noise ratio, 140, 164, 166

matched filter output -, 168

signal-to-quantization-noise ratio, 56

signal-to-shot-noise ratio, 201, 206

signum function, 241

simplex signal set, 163, 164, 227

sinc function, 52, 81, 84, 242

single-input single-output (SISO) systems, 65

smoothing, 175, 176

discrete-time -, 183 spectrum

analyzer, 116

of data signal, 77

of discrete-time process, 57

of filter output, 71

spill-over, 63, 99

split-phase code, 98

spread spectrum, 50

stable system, 87

stationary

points, 233

processes, 9

stochastic processes, 2

continuous -, 4

discrete -, 4, 6

discrete-time -, 4, 5, 31

independent,- 10

spectra of -, 39

strict-sense stationary process, 11

sufficient statistic, 160

superheterodyne, 116

switching center, 208

synchronization, 175

system

causal -, 179

optimal -, 153

stable -, 180

synthesis, 153

Taylor expansion, 237

Thévenin equivalent circuit, 131

thermal noise, 130

current in passive networks, 137

in passive networks, 131

spectrum of current in a resistor, 131

spectrum of voltage across a resistor, 130

voltage in passive networks, 136

time average, 14

transfer function, 67, 89

equivalent baseband -, 104

of bandpass filter, 104

triangular function, 242

trigonometric relations, 231

uncorrelated processes, 27

unipolar return-to-zero, 80

unit-step function, 241 
variance, 26, 55, 197, 201, 204, 240

vector spaces, 215

voice channel, 62

waiting time, 197

white noise, 70, 75, 91, 129, 158, 163, 167, 203, 205, 219

bandpass filtering of -, 110

whitening filter, 169

wide-sense stationary, 219

jointly -, 175 wide-sense stationary processes, 71, 175, 206

definition of -, 12

discrete-time -, 57, 90

jointly -, 20, 70

Wiener filter, 154, 175

discrete time -, 183

Wiener-Hopf equation, 179

Wiener-Khinchin relation, 39

z-transform, 57, 86 
